TRANSACTIONS OF THE

AMERICAN MATHEMATICAL SOCIETY

Volume 363, Number 12, December 2011, Pages 6407-6420

S 0002-9947(2011)05259-1

Article electronically published on July 14, 2011

\title{
ON RELATIVE PROPERTY (T) AND HAAGERUP'S PROPERTY
}

\author{
IONUT CHIFAN AND ADRIAN IOANA
}

\begin{abstract}
We consider the following three properties for countable discrete groups $\Gamma$ : (1) $\Gamma$ has an infinite subgroup with relative property $(T),(2)$ the group von Neumann algebra $L \Gamma$ has a diffuse von Neumann subalgebra with relative property $(\mathrm{T})$ and (3) $\Gamma$ does not have Haagerup's property. It is clear that $(1) \Longrightarrow(2) \Longrightarrow(3)$. We prove that both of the converses are false.
\end{abstract}

\section{INTRODUCTION}

In this paper, we investigate the relationship between relative property $(T)$ and Haagerup's property in the context of countable groups and finite von Neumann algebras. An inclusion $\left(\Gamma_{0} \subset \Gamma\right)$ of countable discrete groups has relative property $(T)$ of Kazhdan-Margulis if any unitary representation of $\Gamma$ which has almost invariant vectors necessarily has a non-zero $\Gamma_{0}$-invariant vector. The classical examples here are $\left(\mathbb{Z}^{2} \subset \mathbb{Z}^{2} \rtimes \mathrm{SL}_{2}(\mathbb{Z})\right)$ and $\left(\mathrm{SL}_{n}(\mathbb{Z}) \subset \mathrm{SL}_{n}(\mathbb{Z})\right)$, for $n \geq 3$ ( $\mathrm{Ka} 67$, Ma82]).

The presence of relative property $(\mathrm{T})$ subgroups is an obstruction to Haagerup's property. A countable group $\Gamma$ is Haagerup if it admits a $c_{0}$ (or mixing) unitary representation which has almost invariant vectors. This class, which includes amenable groups and free groups, is closed under free products and, more surprisingly, wreath products ([CSV09]).

It is clear from the definitions that a group with Haagerup's property cannot have relative property $(\mathrm{T})$ with respect to any infinite subgroup. In CCJJV01, Section 7.1] the authors asked whether the converse holds true, i.e. if having an infinite subgroup with relative property $(\mathrm{T})$ is the only obstruction to Haagerup's property. This question was answered negatively by Y. de Cornulier (Co06a, Co06b $)$. He showed that there are certain groups, e.g. $\Gamma_{\alpha}=\mathbb{Z}[\sqrt[3]{\alpha}]^{3} \rtimes S O_{3}(\mathbb{Z}[\sqrt[3]{\alpha})$, for $\alpha$ not a cube, which do not have relative property (T) with respect to any infinite subgroup but yet have relative property $(\mathrm{T})$ with respect to some infinite subset (see Definition 1.1). The latter property still guarantees the failure of Haagerup's property.

The notion of relative property (T) (or rigidity) for inclusions of finite von Neumann algebras was introduced by S. Popa in Po06a (see Definition 1.4). Since then it has found many striking applications to von Neumann algebras theory and orbit equivalence ergodic theory (see the surveys [Po07] and [Fu09]). Thus, it was used as a key ingredient in Popa's solution to the long-standing problem of finding $\mathrm{II}_{1}$ factors with trivial fundamental group ([Po06a $]$ ). Examples of rigid inclusions

Received by the editors July 14, 2009 and, in revised form, November 23, 2009.

2010 Mathematics Subject Classification. Primary 20F69; Secondary 46L10.

The second author was supported by a Clay Research Fellowship.

(C)2011 American Mathematical Society 
of von Neumann algebras are provided by inclusions of groups. Precisely, the inclusion $\left(\Gamma_{0} \subset \Gamma\right)$ of two countable groups has relative property $(\mathrm{T})$ if and only if the inclusion of group von Neumann algebras $\left(L \Gamma_{0} \subset L \Gamma\right)$ has it ( $\left.\left.\mathrm{Po} 06 \mathrm{a}\right]\right)$. Similarly, there is a notion of Haagerup's property for finite von Neumann algebras which generalizes the corresponding notion for groups (CJ85, Ch83).

Relative property $(\mathrm{T})$ and Haagerup's property are also incompatible in the framework of von Neumann algebras: if a finite von Neumann algebra $N$ has Haagerup's property, then it does not have any diffuse (i.e. non-atomic) relatively rigid subalgebra. The main goal of this paper is to show that the converse of this statement is false even in the case of von Neumann algebras $N$ arising from countable groups. Thus, we are interested in finding a countable group $\Gamma$ such that its group von Neumann algebra $L \Gamma$ neither has Haagerup's property nor any diffuse rigid subalgebra. The natural candidates are Cornulier's examples, $\Gamma_{\alpha}$, since one might hope that the absence of relative property $(\mathrm{T})$ subgroups is inherited by the group von Neumann algebra. However, we prove that this is not the case:

0.1 Theorem (Corollary 2.2). Let $\alpha \in \mathbb{N} \backslash\left\{\beta^{3} \mid \beta \in \mathbb{N}\right\}$ and let $\Gamma_{\alpha}=\mathbb{Z}[\sqrt[3]{\alpha}]^{3} \rtimes$ $\mathrm{SO}_{3}(\mathbb{Z}[\sqrt[3]{\alpha}])$. Then there exists a diffuse von Neumann subalgebra $B$ of $L\left(\mathbb{Z}[\sqrt[3]{\alpha}]^{3}\right)$ such that the inclusion $\left(B \subset L\left(\Gamma_{\alpha}\right)\right)$ is rigid and $B^{\prime} \cap L\left(\Gamma_{\alpha}\right)=L\left(\mathbb{Z}[\sqrt[3]{\alpha}]^{3}\right)$.

In Co06b, a concept of resolutions was introduced in order to quantify the transfer of property (T) from a locally compact group to its lattices. In particular, they can be used to locate the relative property $(\mathrm{T})$ subsets of lattices $\Gamma$ in Lie groups, e.g. $\Gamma=\Gamma_{\alpha}$. The idea behind the proof of Theorem 0.1 is that by combining resolutions with results from [Io09] we can also detect certain rigid subalgebras of $L \Gamma$.

Next, we consider another class of groups. If $A$ and $\Gamma$ are two countable groups and $X$ is a countable $\Gamma$-set, then the generalized wreath product group $A 2_{X} \Gamma$ is defined as $A^{X} \rtimes \Gamma$. If $X=\Gamma$, together with the left multiplication action, then we recover the standard wreath product $A<\Gamma$. Following results from $\mathrm{Po06a}$, Po06b and Io07] we know that if $A$ and $\Gamma$ have Haagerup's property, then the von Neumann algebra $L\left(A \imath_{X} \Gamma\right)$ does not have a diffuse rigid von Neumann subalgebra, regardless of the set $X$. Thus, in order to get examples of groups with the desired properties, it suffices to find a suitable set $X$ for which $A 2_{X} \Gamma$ is not Haagerup.

0.2 Theorem (Corollary 3.4). Let $A$ be a non-trivial countable Haagerup group. Let $\Gamma$ be a countable Haagerup group together with a quotient group $\Gamma_{0}$. Assume that $\Gamma_{0}$ is not Haagerup and endow it with the left multiplication action of $\Gamma$. Then $A 2_{\Gamma_{0}} \Gamma$ is not Haagerup. Thus, the group von Neumann algebra $N=L\left(A 2_{\Gamma_{0}} \Gamma\right)$ does not have Haagerup property and does not admit any diffuse von Neumann subalgebra $B$ such that the inclusion $(B \subset N)$ is rigid.

The proof of Theorem 0.2 is based on a general result: a semidirect product $A \rtimes \Gamma$ is not Haagerup whenever $A$ is abelian, and $\Gamma$ acts on $A$ through a non-Haagerup quotient group, $\Gamma_{0}$.

Recently, Cornulier, Stalder and Valette proved that the class of Haagerup groups is closed under standard wreath products (CSV09). Moreover, they showed that if $A$ and $\Gamma$ are Haagerup groups, then the generalized wreath product $A{ }_{X} \Gamma$ is Haagerup for certain sets $X$, and they conjectured that this is the case for any 
$X$. Theorem 0.2 provides in particular a counterexample to their conjecture. For example, if $\Gamma$ is a free group and $\Gamma_{0}$ is a property (T) quotient of $\Gamma$, then $\mathbb{Z} 2_{\Gamma_{0}} \Gamma$ is not Haagerup.

\section{Preliminaries}

We start this section by reviewing the notion of relative property $(\mathrm{T})$ for groups. Then we explain Cornulier's examples of groups which are not Haagerup but do not have any infinite subgroup with relative property $(\mathrm{T})$. Finally, we recall Popa's notion of rigidity for inclusions of von Neumann algebras.

A continuous unitary representation $\pi$ of a locally compact group $G$ on a Hilbert space $\mathcal{H}$ has almost invariant vectors if for all $\varepsilon>0$ and any compact set $F \subset G$ we can find a unit vector $\xi \in \mathcal{H}$ such that $\|\pi(g)(\xi)-\xi\| \leq \varepsilon$, for all $g \in F$. If $H$ is a closed subgroup of $G$, then the inclusion $(H \subset G)$ has relative property $(\mathrm{T})$ of Kazhdan-Margulis if any unitary representation of $G$ which has almost invariant vectors must have a non-zero $H$-invariant vector ([Ka67, [Ma82]). Also recall that $G$ has Haagerup's property (in short, is Haagerup) if it admits a $c_{0}$ unitary representation $\pi: G \rightarrow \mathcal{U}(\mathcal{H})$ which has almost invariant vectors. Being $c_{0}$ means that for every $\xi, \eta \in \mathcal{H}$ we have that $\lim _{g \rightarrow \infty}\langle\pi(g)(\xi), \eta\rangle=0$.

If a countable, discrete group $\Gamma$ is Haagerup, then it does not have relative property $(\mathrm{T})$ with respect to any infinite subgroup. Cornulier proved that the converse is false (Co06a, Co06b). For example, he showed that if $\alpha \in \mathbb{N}$ $\left\{\beta^{3} \mid \beta \in \mathbb{N}\right\}$, then $\Gamma_{\alpha}=\mathbb{Z}[\sqrt[3]{\alpha}]^{3} \rtimes S O_{3}(\mathbb{Z}[\sqrt[3]{\alpha}])$ neither has Haagerup property nor admits an infinite subgroup with relative property $(\mathrm{T})$. To quickly see that $\Gamma_{\alpha}$ is not Haagerup just notice that it is measure equivalent (see Fu09 for the definition) to the group $\Lambda=\mathbb{Z}^{3} \times\left(\mathbb{Z}[i]^{3} \rtimes S O_{3}(\mathbb{Z}[i])\right)$ which has an infinite subgroup with relative property $(\mathrm{T})$ (i.e. $\left.\mathbb{Z}[i]^{3}\right)$. Indeed, both $\Gamma_{\alpha}$ and $\Lambda$ are lattices in $G=\left(\mathbb{R}^{3} \rtimes S_{3}(\mathbb{R})\right) \times\left(\mathbb{C}^{3} \rtimes S O_{3}(\mathbb{C})\right)($ Ma91, Wi08). This example shows that having an infinite subgroup with relative property $(\mathrm{T})$ is not a measure equivalence invariant. To better explain the failure of Haagerup's property for $\Gamma_{\alpha}$, the following two notions were introduced in $\mathrm{Co06 \textrm {b }}$ :

1.1 Definitions ( $(\mathrm{Co06b})$. (a) Let $p: \Gamma \rightarrow G$ be a morphism between two locally compact groups with dense image. We say that $p$ is a resolution if for any unitary representation $\pi$ of $\Gamma$ which has almost invariant vectors, there exists a subrepresentation $\sigma$ of $\pi$ of the form $\sigma=\tilde{\sigma} \circ p$, where $\tilde{\sigma}$ is a unitary representation of $G$ which has almost invariant vectors.

(b) Given a subset $X$ of a locally compact group $G$, we say that the inclusion $(X \subset G)$ has relative property $(T)$ if for any unitary representation $\pi: G \rightarrow \mathcal{U}(\mathcal{H})$ which has almost invariant vectors and any $\varepsilon>0$, we can find a unit vector $\xi \in \mathcal{H}$ such that $\|\pi(g)(\xi)-\xi\| \leq \varepsilon$, for all $g \in X$.

Resolutions are useful to encode the transfer of relative property (T) from a group to its lattices. To see this, assume that $\Gamma$ is a lattice in a locally compact group $G$, let $H \subset G$ be a normal, closed subgroup such that the inclusion $(H \subset G)$ has relative property (T) and let $p: G \rightarrow G / H$ be the projection. Under these assumptions, [Co6b, Theorem 4.3.1] asserts that the morphism $p_{\mid \Gamma}: \Gamma \rightarrow \overline{p(\Gamma)}$ is a resolution. In the case when $G$ has property (T) and $H=G$, this is just saying that any lattice $\Gamma$ of $G$ has property (T), thus recovering Kazhdan's classical result ([Ka67]). 
Now, if $G=\left(\mathbb{R}^{3} \rtimes S_{3}(\mathbb{R})\right) \times\left(\mathbb{C}^{3} \rtimes S_{3}(\mathbb{C})\right)$ and $H=\mathbb{C}^{3}$, then the inclusion $(H \subset G)$ has relative property $(\mathrm{T})$ (see [Co06b, 3.3.1]). By applying the above theorem to this situation the following was deduced in [Co06b, the proof of 4.6.3]:

1.2 Corollary ([Co6b] $)$. The inclusion $\Gamma_{\alpha} \hookrightarrow \mathbb{R}^{3} \rtimes S O_{3}(\mathbb{Z}[\sqrt[3]{\alpha}])$ is a resolution. Thus, if $\mathcal{B}$ is the unit ball of $\mathbb{R}^{3}$ and $X=\mathbb{Z}[\sqrt[3]{\alpha}]^{3} \cap \mathcal{B}$, then $\left(X \subset \Gamma_{\alpha}\right)$ has relative property $(T)$, for every $\alpha$. In particular, $\Gamma_{\alpha}$ is not Haagerup.

Notice moreover that $X$ is a normal subset of $\Gamma_{\alpha}$. In relation to this, let us note that results from Co06b imply that any lattice in a connected Lie group either has Haagerup's property or admits an infinite, "weakly normal" subset with relative property $(\mathrm{T})$ :

1.3 Corollary. Let $G$ be a connected Lie group which does not have Haagerup's property. Let $\Gamma$ be a lattice in $G$. Then there exists an infinite set $X \subset \Gamma$ such that the inclusion $(X \subset \Gamma)$ has relative property $(T)$ and $\gamma X \gamma^{-1} \cap X$ is infinite, for all $\gamma \in \Gamma$.

Proof. Since $G$ is not Haagerup, by [Co06b, 3.3.1] and [CCJJV01, Chapter 4] we get that it has a non-trivial, normal, closed subgroup $H$ such that the inclusion $(H \subset G$ ) has relative property $(\mathrm{T})$ and $G / H$ has Haagerup's property. Let $p: G \rightarrow$ $G / H$ denote the projection and set $Q=\overline{p(\Gamma)}$. By [Co06b, 4.3.1] the morphism $p_{\mid \Gamma}: \Gamma \rightarrow Q$ is a resolution. Thus, since the inclusion $(\{1\} \subset Q)$ has relative property (T) by [Co06b, 4.2.6], we deduce that the inclusion $((\Gamma \cap H) \subset \Gamma)$ does as well.

Since $\Gamma \cap H$ is a normal subgroup of $\Gamma$ we can hereafter assume that it is finite (otherwise, we can take $X=\Gamma \cap H)$. Under this assumption, we claim that $p(\Gamma)$ is a non-discrete subgroup of $G / H$. Indeed, if $p(\Gamma)$ is discrete, then it must have Haagerup's property, as $G / H$ has. However, $p(\Gamma)$ is isomorphic to $\Gamma /(\Gamma \cap H)$ and, since $\Gamma \cap H$ is finite, we would get that $\Gamma$ is Haagerup, a contradiction.

Next, let $V$ be a neighborhood of $1 \in Q$ with compact closure and define $X=$ $p^{-1}(V) \cap \Gamma$. Since the inclusion $(V \subset Q)$ has relative property $(\mathrm{T})$ by $\mathrm{Co} 06 \mathrm{~b}$, 4.2.6], we deduce that the inclusion $(X \subset \Gamma)$ has relative property $(\mathrm{T})$. To check the normality assertion, fix $\gamma \in \Gamma$ and denote $W=p(\gamma) V p(\gamma)^{-1} \cap V$. Then $\gamma X \gamma^{-1} \cap X=\{x \in \Gamma \mid p(x) \in W\}$. Since $p(\Gamma) \subset G / H$ is non-discrete and $W$ is a neighborhood of $1 \in Q$, the latter set is infinite.

To recall Popa's notion of rigidity for von Neumann algebras, let $M$ be a separable finite von Neumann algebra with a faithful, normal trace $\tau: M \rightarrow \mathbb{C}$ and let $B \subset M$ be a von Neumann subalgebra. A Hilbert space $\mathcal{H}$ is called a Hilbert $M$-bimodule if it admits commuting left and right Hilbert $M$-module structures. A vector $\xi \in \mathcal{H}$ is called tracial if $\langle x \xi, \xi\rangle=\langle\xi x, \xi\rangle=\tau(x)$, for all $x \in M$, and $B$-central if $b \xi=\xi b$, for all $b \in B$. A Hilbert $M$-bimodule $\mathcal{H}$ together with a unit vector $\xi \in \mathcal{H}$ is called a pointed Hilbert $M$-bimodule and is denoted $(\mathcal{H}, \xi)$.

1.4 Definition ( $\mathrm{Po06 \textrm {a }})$. The inclusion $(B \subset M)$ is rigid (or has relative property $(T))$ if for every $\varepsilon>0$ there exists $F \subset M$ finite and $\delta>0$ such that whenever $(\mathcal{H}, \xi)$ is a pointed Hilbert $M$-bimodule with $\xi$ a tracial vector verifying $\|x \xi-\xi x\| \leq \delta$, for all $x \in F$, there exists a $B$-central vector $\eta \in \mathcal{H}$ with $\|\eta-\xi\| \leq \varepsilon$.

The notion of rigidity for inclusions of von Neumann algebras is analogous to and generalizes the notion of relative property $(\mathrm{T})$ for groups. More precisely, given two countable groups $\Gamma_{0} \subset \Gamma$, the inclusion $\left(L\left(\Gamma_{0}\right) \subset L(\Gamma)\right)$ of their group von 
Neumann algebras is rigid if and only if the inclusion $\left(\Gamma_{0} \subset \Gamma\right)$ has relative property (T) ([Po66a, Proposition 5.1]). Now, if $\Gamma$ is a non-amenable subgroup of $\mathrm{SL}_{2}(\mathbb{Z})$ acting on $\mathbb{Z}^{2}$ by matrix multiplication, then the inclusion $\left(\mathbb{Z}^{2} \subset \mathbb{Z}^{2} \rtimes \Gamma\right)$ has relative property $(\mathrm{T})\left(\left[\right.\right.$ Bu91, section 5]), and therefore the inclusion $\left(L\left(\mathbb{Z}^{2}\right) \subset L\left(\mathbb{Z}^{2} \rtimes \Gamma\right)\right)$ is rigid.

The first examples of rigid inclusions of von Neumann algebras which do not rely on relative property $(\mathrm{T})$ for some inclusion of groups have recently been exhibited in $\left\lfloor\right.$ Io09. Thus, it is shown that for any non-amenable subfactor $N$ of $L\left(\mathbb{Z}^{2} \rtimes \mathrm{SL}_{2}(\mathbb{Z})\right)$ which contains $L\left(\mathbb{Z}^{2}\right)$, the inclusion $\left(L\left(\mathbb{Z}^{2}\right) \subset N\right)$ is rigid (โI009, Theorem 3.1]).

\section{Rigid SUbALGEBRAS FROM RESOLUtions}

The main goal of this section is to show that, in certain situations, resolutions can be used to construct rigid subalgebras of von Neumann algebras (Theorem 2.1). Thus, we employ the resolution provided by Corollary 1.2 to deduce that the group von Neumann algebra $L\left(\Gamma_{\alpha}\right)$ has a diffuse rigid subalgebra (Corollary 2.2). This result should be contrasted with the fact that $\Gamma_{\alpha}$ has no infinite subgroup with relative property $(\mathrm{T})$.

2.1 Theorem. Let $\Gamma$ be a countable subgroup of $S O_{n}(\mathbb{R})$, for some $n \geq 3$, and consider the natural action of $\Gamma$ on $H=\mathbb{R}^{n}$.

- Assume that $A \simeq \mathbb{Z}^{m}(m \geq n+1)$ is a $\Gamma$-invariant, dense subgroup of $\mathbb{R}^{n}$. Let $v_{1}, \ldots, v_{m} \in A$ such that $\theta: \mathbb{Z}^{m} \rightarrow A$ given by $\theta\left(\left(x_{i}\right)\right)=\sum_{i=1}^{m} x_{i} v_{i}$ is an isomorphism. Identify the dual $\hat{A}$ of $A$ with $\mathbb{Z}^{m}=\mathbb{T}^{m}=\mathbb{R}^{m} / \mathbb{Z}^{m}$ via the map $\hat{\theta}(\eta)=\eta \circ \theta$.

- Let $p: \mathbb{R}^{n} \rightarrow \mathbb{R}^{m}$ be defined by $p(a)=\left(\left\langle a, v_{1}\right\rangle, \ldots,\left\langle a, v_{m}\right\rangle\right)$, where $\langle.,$.$\rangle is the$ usual scalar product on $\mathbb{R}^{n}$, and denote by $\pi: \mathbb{R}^{m} \rightarrow \mathbb{R}^{m} / p\left(\mathbb{R}^{n}\right)$ the projection.

- Let $i: \mathbb{T}^{m} \rightarrow\left[-\frac{1}{2}, \frac{1}{2}\right)^{m} \subset \mathbb{R}^{m}$ be defined by $i\left(x+\mathbb{Z}^{m}\right)=\left(x+\mathbb{Z}^{m}\right) \cap\left[-\frac{1}{2}, \frac{1}{2}\right)^{m}$, for all $x \in \mathbb{R}^{m}$, and set $q=\pi \circ i: \mathbb{T}^{m} \rightarrow \mathbb{R}^{m} / p\left(\mathbb{R}^{n}\right)$.

Define $(Y, \nu)=\left(q\left(\mathbb{T}^{m}\right), q_{*} \lambda^{m}\right)$, where $\lambda^{m}$ is the Haar measure on $\mathbb{T}^{m}$ and $q_{*} \lambda^{m}$ is the push-forward of $\lambda^{m}$ through $q$. Then we have the following:

(1) $L^{\infty}(Y, \nu)$ is a diffuse von Neumann subalgebra of $L^{\infty}\left(\mathbb{T}^{m}, \lambda^{m}\right)$ (here, we consider the embedding $\left.L^{\infty}(Y, \nu) \ni f \rightarrow f \circ q \in L^{\infty}\left(\mathbb{T}^{m}, \lambda^{m}\right)\right)$.

(2) If the inclusion $A \rtimes \Gamma \rightarrow H \rtimes \Gamma$ is a resolution, then the inclusion of von Neumann algebras $L^{\infty}(Y, \nu) \subset M:=L^{\infty}\left(\mathbb{T}^{m}, \lambda^{m}\right) \rtimes \Gamma$ is rigid.

(3) If $p\left(\mathbb{R}^{n}\right) \cap \mathbb{Z}^{m}=\{0\}$, then $L^{\infty}(Y, \nu)^{\prime} \cap M=L^{\infty}\left(\mathbb{T}^{m}, \lambda^{m}\right)$.

In the statement of this theorem we have used the fact that if $\Gamma$ is a countable group which acts by automorphisms on a countable abelian group $A$, then the action of $\Gamma$ on $\hat{A}$ preserves the Haar measure $h$. Also, we note that the associated crossed product von Neumann algebra $L^{\infty}(\hat{A}, h) \rtimes \Gamma$ is naturally isomorphic to the group von Neumann algebra $L(A \rtimes \Gamma)$ and that this isomorphism identifies $L^{\infty}(\hat{A}, h)$ with $L(A)$.

2.2 Corollary. Let $n \geq 3, \alpha \in \mathbb{N} \backslash\left\{\beta^{3} \mid \beta \in \mathbb{N}\right\}$ and denote $\Gamma_{\alpha}=\mathbb{Z}[\sqrt[3]{\alpha}]^{n} \rtimes$ $S O_{n}(\mathbb{Z}[\sqrt[3]{\alpha}])$. Then there exists a diffuse von Neumann subalgebra $B$ of $L\left(\mathbb{Z}[\sqrt[3]{\alpha}]^{n}\right)$ such that the inclusion $\left(B \subset L\left(\Gamma_{\alpha}\right)\right)$ is rigid and $B^{\prime} \cap L\left(\Gamma_{\alpha}\right)=L\left(\mathbb{Z}[\sqrt[3]{\alpha}]^{n}\right)$. Moreover, if $n=3$, then $L\left(\Gamma_{\alpha}\right)$ is an HT factor in the sense of [Po06a, Definition $6.1]$. 
Proof. For every $(j, k) \in S=\{1, \ldots, n\} \times\{0,1,2\}$, let $v_{j, k} \in \mathbb{R}^{n}$ be given by $v_{j, k}=$ $\left(\alpha^{\frac{k}{3}} \delta_{i, j}\right)_{1 \leq i \leq n}$. Then $\mathbb{Z}[\sqrt[3]{\alpha}]^{n}=\bigoplus_{(j, k) \in S} \mathbb{Z} v_{j, k}$. Denote by $\langle.,$.$\rangle the natural scalar$ product on $\mathbb{R}^{n}$ and let $p: \mathbb{R}^{n} \rightarrow \mathbb{R}^{3 n}=\bigoplus_{(j, k) \in S} \mathbb{R}$ be the homomorphism defined by $p(a)=\left(\left\langle a, v_{j, k}\right\rangle\right)_{j, k}$, for all $a \in \mathbb{R}^{n}$. Explicitly, if $a=\left(a_{i}\right)_{1 \leq i \leq n}$, then $p(a)=$ $\left(a_{j} \alpha^{\frac{k}{3}}\right)_{j, k}$. Since $\alpha^{\frac{1}{3}}$ is irrational, it follows that $p\left(\mathbb{R}^{n}\right) \cap \mathbb{Z}^{3 n}=\{0\}$. Also, by Corollary 1.2 (which holds for every $n \geq 3$, although we only stated it for $n=3$ ) we have that the inclusion $\Gamma_{\alpha} \rightarrow \mathbb{R}^{n} \rtimes S O_{n}(\mathbb{Z}[\sqrt[3]{\alpha}])$ is a resolution. Altogether, Theorem 2.1 gives that there exists a subalgebra $B$ satisfying the conclusion.

Note that $L\left(\mathbb{Z}[\sqrt[3]{\alpha}]^{n}\right)$ is a Cartan subalgebra of $L\left(\Gamma_{\alpha}\right)$. Thus, in view of the first part, in order to show that $L\left(\Gamma_{\alpha}\right)$ is an HT factor, it suffices to argue that $\mathrm{SO}_{3}(\mathbb{Z}[\sqrt[3]{\alpha}])$ has Haagerup's property and that $\Gamma_{\alpha}$ is ICC. The first assertion is a consequence of the following general result: every countable subgroup of $\mathrm{SO}_{3}(\mathbb{R})$ has Haagerup's property (GHW05; see Co06a, Theorem 1.14]).

To prove that $\Gamma_{\alpha}$ is ICC it suffices to show that (1) $\left\{\gamma(a)-a \mid a \in \mathbb{Z}[\sqrt[3]{\alpha}]^{n}\right\}$ is infinite, for every $\gamma \in S O_{n}(\mathbb{Z}[\sqrt[3]{\alpha}]) \backslash\{I\}$ and $(2)\left\{\gamma(a) \mid \gamma \in S O_{n}(\mathbb{Z}[\sqrt[3]{\alpha}])\right\}$ is infinite, for all $a \in \mathbb{Z}[\sqrt[3]{\alpha}]^{n} \backslash\{0\}$. The first assertion is clear since $\mathbb{Z}[\sqrt[3]{\alpha}]^{3}$ is dense in $\mathbb{R}^{3}$. Now, since $S O_{n}(\mathbb{Z}[\sqrt[3]{\alpha}])$ is an irreducible lattice in the semisimple Lie group $S O_{n}(\mathbb{R}) \times S O_{n}(\mathbb{C})$ and $S O_{n}(\mathbb{C})$ is not compact, we deduce that $S O_{n}(\mathbb{Z}[\sqrt[3]{\alpha}])$ is dense in $S O_{n}(\mathbb{R})$ (see e.g. Ma91 or Wi08). This fact implies the second assertion.

For the proof of Theorem 2.1 we need two technical results. To motivate and state the first result, let us fix some notation. For a standard Borel space $X$ (i.e. a Polish space together with its $\sigma$-algebra of Borel subsets) we denote by $\mathcal{M}(X)$ the space of regular Borel probability measures on $X$ and by $B(X)$ the algebra of bounded Borel complex-valued functions on $X$. Given two measures $\mu, \nu \in \mathcal{M}(X)$, the norm $\|\mu-\nu\|$ is equal to $\sup _{f \in B(X),\|f\|_{\infty} \leq 1}\left|\int_{X} f d \mu-\int_{X} f d \nu\right|$.

Now, if an inclusion of the form $(A \subset A \rtimes \Gamma$ ) (where $\Gamma$ is a countable group acting by automorphisms on a countable abelian group $A$ ) has relative property (T), then any sequence of measures $\mu_{n} \in \mathcal{M}(\hat{A})$ which converge weakly to $\delta_{1}$ and are almost $\Gamma$-invariant must "concentrate" at the identity element $1 \in \hat{A}$, i.e. $\lim _{n \rightarrow \infty} \mu_{n}(\{1\})=0$ ([o09, Theorem 5.1]; the converse is also true; see [Bu91]). The next proposition roughly asserts that the presence of certain resolutions for $A \rtimes \Gamma$ also guarantees that almost invariant measures on $\hat{A}$ concentrate on some subsets.

2.3 Proposition. Let $H$ be a locally compact abelian group together with a dense countable subgroup $A$, and denote by $p: \hat{H} \rightarrow \hat{A}$ the map induced by restricting characters. Let $\Gamma$ be a countable group which acts by automorphisms on $H$ and leaves $A$ invariant. Suppose that the inclusion $A \rtimes \Gamma \rightarrow H \rtimes \Gamma$ is a resolution. Also, let $V \subset \hat{H}$ be a $\Gamma$-invariant neighborhood of $1 \in \hat{H}$.

Then for any sequence of measures $\mu_{n} \in \mathcal{M}(\hat{A})$ which converge weakly to $\delta_{1}$ and satisfy $\lim _{n \rightarrow \infty}\left\|\gamma_{*} \mu_{n}-\mu_{n}\right\|=0$, for all $\gamma \in \Gamma$, we have that $\lim _{n \rightarrow \infty} \mu_{n}(p(V))=1$. In particular, we have that $\lim _{n \rightarrow \infty} \mu_{n}(p(\hat{H}))=1$.

Proof. Given $V \subset \hat{H}$ and a sequence $\left\{\mu_{n}\right\}_{n \geq 1} \subset \mathcal{M}(\hat{A})$ as in the hypothesis, we begin by showing:

Claim. There exists $n$ such that $\mu_{n}(p(V))>0$.

Proof of the Claim. Let us first prove the Claim under the additional assumption that $\mu_{n}$ is $\Gamma$-quasi-invariant, for all $n$. Fix $n \geq 1$. Since $\mu_{n}$ is $\Gamma$-quasi-invariant, 
we can define $g_{\gamma}=\left(d\left(\gamma_{*} \mu_{n}\right) / d \mu_{n}\right)^{\frac{1}{2}}$, for all $\gamma \in \Gamma$, where $d\left(\gamma_{*} \mu_{n}\right) / d \mu_{n}$ denotes the Radon-Nikodym derivative of $\gamma_{*} \mu_{n}$ with respect to $\mu_{n}$. Next, we see every $a \in A$ as a character on $\hat{A}$ and therefore as a function in $L^{\infty}\left(\hat{A}, \mu_{n}\right)$. Then the formulas

$$
\pi_{n}(a)(f)=a f, \pi_{n}(\gamma)(f)=g_{\gamma}\left(f \circ \gamma^{-1}\right)
$$

for all $a \in A, \gamma \in \Gamma$ and $f \in L^{2}\left(\hat{A}, \mu_{n}\right)$, define a unitary representation $\pi_{n}: A \rtimes \Gamma \rightarrow$ $\mathcal{U}\left(L^{2}\left(\hat{A}, \mu_{n}\right)\right)$. Let $\xi_{n}=1_{\hat{A}} \in L^{2}\left(\hat{A}, \mu_{n}\right)$. For all $a \in A$ and $\gamma \in \Gamma$, we have that

$$
\begin{aligned}
& \left\|\pi_{n}(\gamma)\left(\xi_{n}\right)-\xi_{n}\right\|=\left\|g_{\gamma}-1\right\|_{2} \leq\left\|g_{\gamma}^{2}-1\right\|_{1}^{\frac{1}{2}}=\left\|\gamma_{*} \mu_{n}-\mu_{n}\right\|^{\frac{1}{2}} \\
& \left\|\pi_{n}(a)\left(\xi_{n}\right)-\xi_{n}\right\|=\left(\int_{\hat{A}}|\eta(a)-1|^{2} d \mu_{n}(\eta)\right)^{\frac{1}{2}}
\end{aligned}
$$

Using the assumptions made on $\mu_{n}$, it follows that the vectors $\xi_{n}$ form an almost invariant sequence for the representation $\pi=\bigoplus_{n \geq 1} \pi_{n}: A \rtimes \Gamma \rightarrow \mathcal{U}(\mathcal{H})$, where $\mathcal{H}=$ $\bigoplus_{n \geq 1} L^{2}\left(\hat{A}, \mu_{n}\right)$. Since the inclusion $A \rtimes \Gamma \rightarrow H \rtimes \Gamma$ is a resolution, we can find a $\pi$-invariant Hilbert subspace $\mathcal{K} \subset \mathcal{H}$ and a unitary representation $\sigma: H \rtimes \Gamma \rightarrow \mathcal{U}(\mathcal{K})$ which has almost invariant vectors and satisfies $\sigma(g)=\pi(g)_{\mid \mathcal{K}}$, for all $g \in A \rtimes \Gamma$. Let $\left\{\zeta_{k}\right\}_{k \geq 1} \subset \mathcal{K}$ be a sequence of $\sigma$-almost invariant unit vectors. For every $k$, let $\nu_{k} \in \mathcal{M}(\hat{\hat{H}})$ be given by $\left\langle\sigma(g) \zeta_{k}, \zeta_{k}\right\rangle=\int_{\hat{H}} \eta(g) d \nu_{k}(\eta)$, for all $g \in H$. Notice that $\nu_{k}$ converge weakly to $\delta_{1}$, as $k \rightarrow \infty$.

Next, if we set $\rho_{k}=p_{*} \nu_{k} \in \mathcal{M}(\hat{A})$, then for each $a \in A$ we have that

$$
\begin{gathered}
\int_{\hat{A}} a d \rho_{k}=\int_{\hat{H}}(a \circ p) d \nu_{k}=\int_{\hat{H}} \eta(a) d \nu_{k}(\eta) \\
\left\langle\sigma(a) \zeta_{k}, \zeta_{k}\right\rangle=\left\langle\pi(a)\left(\zeta_{k}\right), \zeta_{k}\right\rangle .
\end{gathered}
$$

Now, for every $k \geq 1$, decompose $\zeta_{k}=\sum_{n \geq 1} \zeta_{k}^{n}$, where $\zeta_{k}^{n} \in L^{2}\left(\hat{A}, \mu_{n}\right)$. Thus, for all $a \in A$, we have that

$$
\left\langle\pi(a)\left(\zeta_{k}\right), \zeta_{k}\right\rangle=\sum_{n \geq 1} \int_{\hat{A}} a\left|\zeta_{k}^{n}\right|^{2} d \mu_{n}
$$

By combining (a) and (b) we deduce that $d \rho_{k}=\sum_{n \geq 1}\left|\zeta_{k}^{n}\right|^{2} d \mu_{n}$, for all $k \geq 1$. Since $\rho_{k}(p(V))=\nu_{k}(V)$ and $\nu_{k} \rightarrow \delta_{1}$ weakly, we get that $\lim _{k \rightarrow \infty} \rho_{k}(p(V))=1$. Thus, we can find $n$ such that $\mu_{n}(p(V))>0$.

In general, if $\mu_{n}$ are not necessarily quasi-invariant, let $\left\{\gamma_{i}\right\}_{i \geq 1}$ be an enumeration of $\Gamma$. For every $n$, set $\mu_{n}^{\prime}=\sum_{i \geq 1} \frac{1}{2^{i}} \gamma_{i_{*}} \mu_{n}$. Then $\mu_{n}^{\prime}$ are $\Gamma$ quasi-invariant measures which satisfy the hypothesis. By applying the first part of the proof, we get that $\mu_{n}(p(V))=\mu_{n}^{\prime}(p(V))>0$, for some $n$.

Suppose by contradiction that the conclusion of the theorem is false. Then, after passing to a subsequence, we can assume that $\lim _{n \rightarrow \infty} \mu_{n}(p(V))=c<1$. Thus, for large enough $n$ we have that $\mu_{n}(\hat{A} \backslash p(V))>0$, so can define $\mu_{n}^{\prime} \in \mathcal{M}(\hat{A})$ by letting $\mu_{n}^{\prime}(X)=\frac{\mu_{n}(X \backslash p(V))}{\mu_{n}(\hat{A} \backslash p(V))}$, for every Borel set $X \subset \hat{A}$. Notice that $\mu_{n}^{\prime} \rightarrow \delta_{1}$ weakly, as $n \rightarrow \infty$. To see this, just remark that for every neighborhood $W$ of $1 \in \hat{A}$ we have that $\mu_{n}^{\prime}(\hat{A} \backslash W) \leq \frac{\mu_{n}(\hat{A} \backslash W)}{\mu_{n}(\hat{A} \backslash p(V))} \rightarrow 0$, as $n \rightarrow \infty$.

Next, it is easy to see that since $V$ is $\Gamma$-invariant, we get that

$$
\lim _{n \rightarrow \infty}\left\|\gamma_{*} \mu_{n}^{\prime}-\mu_{n}^{\prime}\right\|=0
$$


for all $\gamma \in \Gamma$. Altogether, it follows that $\mu_{n}^{\prime}$ satisfy the conditions of the hypothesis. Therefore, we can apply the claim and derive that $\mu_{n}^{\prime}(p(V))>0$, for some $n$, a contradiction.

The second ingredient needed in the proof of Theorem 2.1 is the following criterion for rigidity which we derive as a consequence of results from [o09].

2.4 Proposition. Let $\Gamma \curvearrowright(X, \mu)$ be a measure preserving action of a countable group $\Gamma$ on a standard probability space $(X, \mu)$. Let $p^{i}: X \times X \rightarrow X$ be the projection $p^{i}\left(x_{1}, x_{2}\right)=x_{i}$, for $i \in\{1,2\}$, and endow $X \times X$ with the diagonal action of $\Gamma$. Let $(Y, \nu)$ be another probability space together with a measurable, measure preserving onto map $q: X \rightarrow Y$. View $L^{\infty}(Y, \nu)$ as a von Neumann subalgebra of $L^{\infty}(X, \mu)$ via the embedding $L^{\infty}(Y, \nu) \ni f \rightarrow f \circ q \in L^{\infty}(X, \mu)$.

Assume that for any sequence of measures $\left\{\nu_{n}\right\}_{n \geq 1} \subset \mathcal{M}(X \times X)$ such that $p_{*}^{i}\left(\nu_{n}\right)=\mu$, for all $i \in\{1,2\}$ and $n \geq 1$,

(i) $\lim _{n \rightarrow \infty} \int_{X \times X} f_{1}(x) f_{2}(y) d \nu_{n}(x, y)=\int_{X} f_{1} f_{2} d \mu$, for all $f_{1}, f_{2} \in B(X)$, and

(ii) $\lim _{n \rightarrow \infty}\left\|\gamma_{*} \nu_{n}-\nu_{n}\right\|=0$, for all $\gamma \in \Gamma$; we have that

$$
\lim _{n \rightarrow \infty} \nu_{n}(\{(x, y) \in X \times X \mid q(x)=q(y)\})=1 .
$$

Then the inclusion of von Neumann algebras $L^{\infty}(Y, \nu) \subset L^{\infty}(X, \mu) \rtimes \Gamma$ is rigid.

Proof. Denote $M=L^{\infty}(X, \mu) \rtimes \Gamma$ and let $\left(\mathcal{H}_{n}, \xi_{n}\right)$ be a sequence of pointed Hilbert $M$-bimodules such that $\lim _{n \rightarrow \infty}\left\|z \xi_{n}-\xi_{n} z\right\|=0$, for all $z \in M$. To get the conclusion we have to show that there exists a sequence $\eta_{n} \in \mathcal{H}_{n}$ of $L^{\infty}(Y, \nu)$ central vectors such that $\lim _{n \rightarrow \infty}\left\|\eta_{n}-\xi_{n}\right\|=0$ (see Definition 1.4).

By [Io09, Lemma 2.1] we can find a sequence $\left\{\nu_{n}\right\}_{n \geq 1} \subset \mathcal{M}(X \times X)$ which verifies all the conditions from the hypothesis and satisfies $\int_{X \times X} f_{1}(x) f_{2}(y) d \nu_{n}(x, y)=$ $\left\langle f_{1} \xi_{n} f_{2}, \xi_{n}\right\rangle$, for all $f_{1}, f_{2} \in B(X)$. Thus, if $\Delta_{q}:=\{(x, y) \in X \times X \mid q(x)=q(y)\}$, then $\lim _{n \rightarrow \infty} \nu_{n}\left(\Delta_{q}\right)=1$.

Next, for every $f_{1}, f_{2} \in B(X)$, let $f_{1} \otimes f_{2}: X \times X \rightarrow \mathbb{C}$ be given by $\left(f_{1} \otimes\right.$ $\left.f_{2}\right)(x, y)=f_{1}(x) f_{2}(y)$. Then notice that by the way $\nu_{n}$ is defined, the map $L^{2}\left(X \times X, \nu_{n}\right) \ni f_{1} \otimes f_{2} \rightarrow f_{1} \xi_{n} f_{2} \in \mathcal{H}_{n}$, for every $f_{1}, f_{2} \in B(X)$, extends to an embedding of Hilbert $L^{\infty}(X, \mu)$-bimodules $\theta_{n}: L^{2}\left(X \times X, \nu_{n}\right) \rightarrow \mathcal{H}_{n}$. Here, on $L^{2}\left(X \times X, \nu_{n}\right)$, we consider the $L^{\infty}(X, \mu)$-bimodule structure given by $f_{1}$. $g \cdot f_{2}=\left(f_{1} \otimes f_{2}\right) g$, for all $f_{1}, f_{2} \in L^{\infty}(X, \mu)$ and $g \in L^{2}\left(X \times X, \nu_{n}\right)$. Let $\eta_{n}=\theta_{n}\left(1_{\Delta_{q}}\right)$. Since $1_{\Delta_{q}} \in L^{2}\left(X \times X, \nu_{n}\right)$ is an $L^{\infty}(Y, \nu)$-central vector, we get that $\eta_{n} \in \mathcal{H}_{n}$ is an $L^{\infty}(Y, \nu)$-central vector. Finally, notice that $\left\|\eta_{n}-\xi_{n}\right\|=$ $\left\|1_{\Delta_{q}}-1_{X \times X}\right\|_{L^{2}\left(X \times X, \nu_{n}\right)}=\sqrt{\nu_{n}\left((X \times X) \backslash \Delta_{q}\right)} \rightarrow 0$, as $n \rightarrow \infty$.

We are now ready to prove Theorem 2.1:

Proof of Theorem 2.1. (1) To derive that $L^{\infty}(Y, \nu)$ is diffuse, we only need to show that $\lambda^{m}\left(q^{-1}(\{y\})\right)=0$, for every $y \in Y$. This is clear since $n<m$ and $q^{-1}(\{y\}) \subset$ $\left(y+p\left(\mathbb{R}^{n}\right)\right)+\mathbb{Z}^{m} \subset \mathbb{T}^{m}$.

(2) To prove the rigidity assertion, let $\nu_{k} \in \mathcal{M}\left(\mathbb{T}^{m} \times \mathbb{T}^{m}\right)$ be a sequence of measures such that

$$
\lim _{k \rightarrow \infty} \int_{\mathbb{T}^{m} \times \mathbb{T}^{m}} f_{1}(x) f_{2}(y) d \nu_{k}(x, y)=\int_{\mathbb{T}^{m}} f_{1} f_{2} d \lambda^{m}, \forall f_{1}, f_{2} \in B\left(\mathbb{T}^{m}\right)
$$


and

$$
\lim _{k \rightarrow \infty}\left\|\gamma_{*} \nu_{k}-\nu_{k}\right\|=0, \forall \gamma \in \Gamma
$$

Denote $\Delta_{q}=\left\{(x, y) \in \mathbb{T}^{m} \times \mathbb{T}^{m} \mid q(x)=q(y)\right\}$. Following Proposition 2.4 in order to get the conclusion, it suffices to argue that $\lim _{k \rightarrow \infty} \nu_{k}\left(\Delta_{q}\right)=1$. To this end, notice that (a) gives that for every bounded Borel function $f$ on $\mathbb{T}^{m}$ we have that $\lim _{k \rightarrow \infty} \int_{\mathbb{T}^{m} \times \mathbb{T}^{m}}|f(x)-f(y)|^{2} d \nu_{k}(x, y)=0$. This implies that

$$
\lim _{k \rightarrow \infty} \int_{\mathbb{T}^{m} \times \mathbb{T}^{m}}\|i(x)-i(y)\|^{2} d \nu_{k}(x, y)=0,
$$

where $\|$.$\| denotes the Euclidian norm on \mathbb{R}^{m}$. Thus, we deduce that

$$
\lim _{k \rightarrow \infty} \nu_{k}\left(\left\{(x, y) \in \mathbb{T}^{m} \times \mathbb{T}^{m} \mid\|i(x)-i(y)\| \leq \frac{1}{2}\right\}\right)=1 .
$$

Next, assume that $x, y \in \mathbb{T}^{m}$ satisfy $\|i(x)-i(y)\| \leq \frac{1}{2}$. Since $i(x)-i(y) \in(x-y)+$ $\mathbb{Z}^{m}$, we deduce that $i(x)-i(y)=i(x-y)$ and therefore that $q(x)-q(y)=q(x-y)$. By combining this fact with (c), we get that

$$
\lim _{k \rightarrow \infty} \nu_{k}\left(\left\{(x, y) \in \mathbb{T}^{m} \times \mathbb{T}^{m} \mid q(x)-q(y)=q(x-y)\right\}\right)=1 .
$$

Thus, showing that $\lim _{k \rightarrow \infty} \nu_{k}\left(\Delta_{q}\right)=1$ is equivalent to proving that

$$
\lim _{k \rightarrow \infty} \nu_{k}\left(\left\{(x, y) \in \mathbb{T}^{m} \times \mathbb{T}^{m} \mid q(x-y)=0\right\}\right)=1 .
$$

If we let $r: \mathbb{T}^{m} \times \mathbb{T}^{m} \rightarrow \mathbb{T}^{m}$ be given by $r(x, y)=x-y$ and, for every $k$, define $\mu_{k}=r_{*} \nu_{k} \in \mathcal{M}\left(\mathbb{T}^{m}\right)$, then (d) can be rewritten as

$$
\lim _{k \rightarrow \infty} \mu_{k}\left(\left\{x \in \mathbb{T}^{m} \mid q(x)=0\right\}\right)=1 .
$$

Now, note that the inclusion $A=\mathbb{Z} v_{1} \oplus \ldots \oplus \mathbb{Z} v_{m} \subset H=\mathbb{R}^{n}$ gives rise to a homomorphism $p^{\prime}: \hat{H} \simeq \mathbb{R}^{n} \rightarrow \hat{A} \simeq \mathbb{T}^{m}$ given by $p^{\prime}(a)=\left(\left\langle a, v_{1}\right\rangle+\mathbb{Z}, \ldots,\left\langle a, v_{m}\right\rangle+\mathbb{Z}\right)$, for all $a \in \mathbb{R}^{n}$. In other words, $p^{\prime}$ is the composition between $p$ and the projection $\mathbb{R}^{m} \rightarrow \mathbb{T}^{m}$. Let $\varepsilon>0$ such that $V=\left\{a \in \mathbb{R}^{n} \mid\|a\| \leq \varepsilon\right\}$ satisfies $p(V) \subset\left[-\frac{1}{2}, \frac{1}{2}\right)^{m}$. We claim that $p^{\prime}(V) \subset\left\{x \in \mathbb{T}^{m} \mid q(x)=0\right\}$. Indeed, if $a \in V$, then $p(a) \in\left[-\frac{1}{2}, \frac{1}{2}\right)^{m}$. Thus $i\left(p^{\prime}(a)\right)=p(a)$, and hence $q\left(p^{\prime}(a)\right)=(\pi \circ i)\left(p^{\prime}(a)\right)=\pi(p(a))=0$.

On the other hand, $V$ is a $\Gamma$-invariant neighborhood of $0 \in \mathbb{R}^{n}$. Also, remark that (a) implies that $\mu_{k}$ converge weakly to $\delta_{0}$ while (b) implies that

$$
\lim _{k \rightarrow \infty}\left\|\gamma_{*} \mu_{k}-\mu_{k}\right\|=0
$$

for all $\gamma \in \Gamma$. By applying Proposition 2.3 we deduce that $\lim _{k \rightarrow \infty} \mu_{k}\left(p^{\prime}(V)\right)=1$. Since $p^{\prime}(V) \subset\left\{x \in \mathbb{T}^{m} \mid q(x)=0\right\}$, we get that $\lim _{k \rightarrow \infty} \mu_{k}\left(\left\{x \in \mathbb{T}^{m} \mid q(x)=0\right\}\right)=1$. This proves $(\mathrm{d})$ and thus the conclusion.

(3) First, it is easy to see that the conclusion is equivalent to

$$
\lambda^{m}\left(\left\{x \in \mathbb{T}^{m} \mid q(\gamma x)=q(x)\right\}\right)=0,
$$

for all $\gamma \in \Gamma \backslash\{I\}$ (where $I$ denotes the identity matrix). Assuming that this is not the case we can find $\gamma \in \Gamma \backslash\{I\}$ such that $\lambda^{m}\left(\left\{x \in \mathbb{T}^{m} \mid q(\gamma x)=q(x)\right\}\right)>0$. By using the definition of $q$, this implies that

$$
\lambda^{m}\left(\left\{x \in \mathbb{T}^{m} \mid \gamma x-x \in p\left(\mathbb{R}^{n}\right)+\mathbb{Z}^{m}\right\}\right)>0 .
$$


Second, notice that the action of $\Gamma$ on $\mathbb{Z}^{m} \simeq A$ is realized through a homomorphism $\rho: \Gamma \rightarrow \mathrm{GL}_{m}(\mathbb{Z})$. The (dual) action of $\Gamma$ on $\mathbb{T}^{m} \simeq \hat{A}$ is then given by $\gamma\left(x+\mathbb{Z}^{m}\right)=$ $\left(\rho(\gamma)^{-1}\right)^{t}(x)+\mathbb{Z}^{m}$. Altogether, (e) implies that if $\mu^{m}$ denotes the Lebesgue measure on $\mathbb{R}^{m}$, then $\mu^{m}\left(\left\{x \in \mathbb{R}^{m} \mid\left(\rho(\gamma)^{-1}\right)^{t}(x)-(x) \in p\left(\mathbb{R}^{n}\right)+\mathbb{Z}^{m}\right\}\right)>0$. This easily implies that

$$
\left(\rho\left(\gamma^{-1}\right)^{t}-I\right)\left(\mathbb{R}^{m}\right) \subset p\left(\mathbb{R}^{n}\right) .
$$

Finally, since $\rho\left(\gamma^{-1}\right) \in \mathrm{GL}_{m}(\mathbb{Z})$, we get that $\left(\rho\left(\gamma^{-1}\right)^{t}-I\right)\left(\mathbb{Z}^{m}\right) \subset \mathbb{Z}^{m}$. By combining this with (f) and the fact that $p\left(\mathbb{R}^{n}\right) \cap \mathbb{Z}^{m}=\{0\}$, we deduce that $\left(\rho\left(\gamma^{-1}\right)^{t}-I\right)\left(\mathbb{Z}^{m}\right)=\{0\}$. This means that $\rho(\gamma)=I$, or, equivalently, that $\gamma$ acts trivially on $A$. Since $A$ is dense in $\mathbb{R}^{n}$, we further get that $\gamma$ acts trivially on $\mathbb{R}^{n}$ which implies that $\gamma=I$, a contradiction.

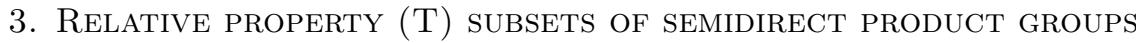

In this section we show that Haagerup's property is not preserved under generalized wreath products. Using this fact we give the first examples of von Neumann algebras which neither have Haagerup's property nor admit any diffuse rigid von Neumann subalgebras. We start with the following result which asserts that if a group $\Gamma$ acts on an abelian group $A$ through a quotient group $\Gamma_{0}$, then the presence of a relative property $(\mathrm{T})$ subset in $\Gamma_{0}$ (or the lack of Haagerup's property) is inherited by the semidirect product $A \rtimes \Gamma$.

3.1 Theorem. Let $\Gamma_{0}$ be a countable group and let $\rho: \Gamma_{0} \rightarrow$ Aut $(A)$ be an action by automorphisms on a countable abelian group $A$. Let $\Gamma$ be a countable group together with a surjective homomorphism $p: \Gamma \rightarrow \Gamma_{0}$, and consider the action of $\Gamma$ on $A$ given by $\tilde{\rho}=\rho \circ p: \Gamma \rightarrow A u t(A)$.

(1) Suppose that $X$ is a subset of $\Gamma_{0}$ such that the inclusion $\left(X \subset \Gamma_{0}\right)$ has relative property (T). For $a \in A$, let $X_{a}=\{\rho(\gamma)(a) \mid \gamma \in X\} \subset A$. Then the semidirect product $A \rtimes_{\tilde{\rho}} \Gamma$ has relative property $(T)$ with respect to $X_{a}$, for every $a \in A$.

(2) Assume that there is $a \in A$ such that its stabilizer $\left\{\gamma \in \Gamma_{0} \mid \rho(\gamma)(a)=a\right\}$ in $\Gamma_{0}$ is finite. If $\Gamma_{0}$ does not have Haagerup's property, then $A \rtimes_{\tilde{\rho}} \Gamma$ does not have Haagerup's property.

Proof. (1) Fix $a \in A$ and let $\pi: A \rtimes_{\tilde{\rho}} \Gamma \rightarrow \mathcal{U}(\mathcal{H})$ be a unitary representation which admits a sequence $\left\{\xi_{n}\right\}_{n \geq 1} \subset \mathcal{H}$ of almost invariant, unit vectors. To get the conclusion we have to show that $\xi_{n}$ are uniformly $\pi\left(X_{a}\right)$-almost invariant, i.e. $\lim _{n \rightarrow \infty} \sup _{\gamma \in X_{a}}\left\|\pi(\rho(\gamma)(a))\left(\xi_{n}\right)-\xi_{n}\right\|=0$.

First, for every $n \geq 1$, let $\mu_{n} \in \mathcal{M}(\hat{A})$ such that $\left\langle\pi(a) \xi_{n}, \xi_{n}\right\rangle=\int_{\hat{A}} a d \mu_{n}$, for each $a \in A$. By the proof of [Bu91, Proposition 7] we have that $\left\|\gamma_{*} \mu_{n}-\mu_{n}\right\| \leq$ $2\left\|\pi(\gamma)\left(\xi_{n}\right)-\xi_{n}\right\|$, for all $\gamma \in \Gamma$. Here, on $\hat{A}$ we consider the natural actions of $\Gamma$ and $\Gamma_{0}$ induced by $\tilde{\rho}$ and $\rho$, respectively. For every $\gamma \in \Gamma_{0}$, fix $\tilde{\gamma} \in \Gamma$ such that $p(\tilde{\gamma})=\gamma$. Then the above implies that

$$
\left\|\gamma_{*} \mu_{n}-\mu_{n}\right\|=\left\|\tilde{\gamma}_{*} \mu_{n}-\mu_{n}\right\| \leq 2\left\|\pi(\tilde{\gamma})\left(\xi_{n}\right)-\xi_{n}\right\|, \forall \gamma \in \Gamma_{0}
$$

Second, let $\left\{\gamma_{i}\right\}_{i \geq 1}$ be an enumeration of $\Gamma_{0} \backslash\{e\}$. For every $n \geq 1$, let $\nu_{n} \in \mathcal{M}(\hat{A})$ be given by $\nu_{n}=\left(1-\frac{1}{2^{n}}\right) \mu_{n}+\sum_{i \geq 1} \frac{1}{2^{i+n}} \gamma_{i_{*}} \mu_{n}$. Then we have that $\left\|\nu_{n}-\mu_{n}\right\| \leq \frac{1}{2^{n-1}}$, 
for all $n \geq 1$, and thus (a) implies that

$$
\begin{gathered}
\left\|\gamma_{*} \nu_{n}-\nu_{n}\right\| \leq 2\left\|\nu_{n}-\mu_{n}\right\|+\left\|\gamma_{*} \mu_{n}-\mu_{n}\right\| \\
\leq \frac{1}{2^{n-2}}+2\left\|\pi(\tilde{\gamma})\left(\xi_{n}\right)-\xi_{n}\right\|, \forall \gamma \in \Gamma_{0} .
\end{gathered}
$$

Next, fix $n \geq 1$. Notice that $\nu_{n}$ is a $\Gamma_{0}$-quasi-invariant measure and let $g_{\gamma}=$ $\left(d\left(\gamma_{*} \nu_{n}\right) / d \nu_{n}\right)^{\frac{1}{2}} \in L^{2}\left(\hat{A}, \nu_{n}\right)$, for every $\gamma \in \Gamma_{0}$. The formula $\sigma_{n}(\gamma)(f)=g_{\gamma}\left(f \circ \gamma^{-1}\right)$, for all $f \in L^{2}\left(\hat{A}, \nu_{n}\right)$ and $\gamma \in \Gamma_{0}$, defines a unitary representation $\sigma_{n}: \Gamma_{0} \rightarrow$ $\mathcal{U}\left(L^{2}\left(\hat{A}, \nu_{n}\right)\right)$. If $\eta_{n}=1_{\hat{A}} \in L^{2}\left(\hat{A}, \nu_{n}\right)$, then, as in the proof of Proposition 2.3, we have that

$$
\left\|\sigma_{n}(\gamma)\left(\eta_{n}\right)-\eta_{n}\right\| \leq\left\|\gamma_{*} \nu_{n}-\nu_{n}\right\|^{\frac{1}{2}}, \forall \gamma \in \Gamma_{0} .
$$

Since the vectors $\xi_{n}$ are $\pi(\Gamma)$-almost invariant, by combining (b) and (c) we deduce that $\lim _{n \rightarrow \infty}\left\|\sigma_{n}(\gamma)\left(\eta_{n}\right)-\eta_{n}\right\|=0$, for each $\gamma \in \Gamma_{0}$. Since the inclusion $\left(X \subset \Gamma_{0}\right.$ ) has relative property $(\mathrm{T})$, by [Co6b, Theorem 1.1] we get that $\varepsilon_{n}:=$ $\sup _{\gamma \in X}\left\|\sigma_{n}(\gamma)\left(\eta_{n}\right)-\eta_{n}\right\| \rightarrow 0$, as $n \rightarrow \infty$. Now, for $\gamma \in X$ we have that

$$
\begin{aligned}
\left\|\gamma_{*} \nu_{n}-\nu_{n}\right\| & =\left\|g_{\gamma}^{2}-1\right\|_{1} \leq\left\|g_{\gamma}+1\right\|_{2}\left\|g_{\gamma}-1\right\|_{2} \leq 2\left\|g_{\gamma}-1\right\|_{2} \\
& =2\left\|\sigma_{n}(\gamma)\left(\eta_{n}\right)-\eta_{n}\right\| \leq 2 \varepsilon_{n}, \forall n \geq 1 .
\end{aligned}
$$

Thus, we get that $\left\|\gamma_{*} \mu_{n}-\mu_{n}\right\| \leq 2|| \nu_{n}-\mu_{n}\|+\| \gamma_{*} \nu_{n}-\nu_{n} \| \leq \frac{1}{2^{n-1}}+2 \varepsilon_{n}$, for all $\gamma \in X$. This implies that for every $\gamma \in X$ we have that

$$
\begin{gathered}
\left|\left\langle\pi(\rho(\gamma)(a))\left(\xi_{n}\right), \xi_{n}\right\rangle-\left\langle\pi(a)\left(\xi_{n}\right), \xi_{n}\right\rangle\right|=\left|\int_{\hat{A}} \rho(\gamma)(a) d \mu_{n}-\int_{\hat{A}} a d \mu_{n}\right| \\
=\left|\int_{\hat{A}}\left(a \circ \gamma^{-1}\right) d \mu_{n}-\int_{\hat{A}} a d \mu_{n}\right| \leq|| \gamma_{*} \mu_{n}-\mu_{n}|| \leq \frac{1}{2^{n-1}}+2 \varepsilon_{n} .
\end{gathered}
$$

Finally, (d) together with a standard calculation gives that

$$
\left\|\pi(\rho(\gamma)(a))\left(\xi_{n}\right)-\xi_{n}\right\|^{2} \leq\left\|\pi(a)\left(\xi_{n}\right)-\xi_{n}\right\|^{2}+2\left(\frac{1}{2^{n-1}}+2 \varepsilon_{n}\right), \forall \gamma \in X,
$$

which proves the conclusion.

(2) Assume by contradiction that $A \rtimes_{\tilde{\rho}} \Gamma$ has Haagerup's property, while $\Gamma_{0}$ does not have it. Thus we can find a $c_{0}$ unitary representation $\pi: A \rtimes_{\tilde{\rho}} \Gamma \rightarrow \mathcal{U}(\mathcal{H})$ which admits a sequence $\left\{\xi_{n}\right\}_{n>1} \subset \mathcal{H}$ of almost invariant unit vectors. Let $\sigma_{n}: \Gamma_{0} \rightarrow$ $\mathcal{U}\left(L^{2}\left(\hat{A}, \nu_{n}\right)\right)$ and $\eta_{n} \in L^{2}\left(\hat{A}, \nu_{n}\right)$ be constructed as in the proof of part (1). Recall that $\eta_{n}$ are almost invariant unit vectors, i.e. $\lim _{n \rightarrow \infty}\left\|\sigma_{n}(\gamma)\left(\eta_{n}\right)-\eta_{n}\right\|=0$, for each $\gamma \in \Gamma_{0}$.

Since $\Gamma_{0}$ does not have Haagerup's property, by [Pe09, Theorem 2.6] we can find an infinite subset $X$ of $\Gamma_{0}$ and an increasing sequence $\left\{k_{n}\right\}_{n \geq 1}$ of natural numbers such that $\lim _{n \rightarrow \infty} \sup _{\gamma \in X}\left\|\sigma_{k_{n}}(\gamma)\left(\eta_{k_{n}}\right)-\eta_{k_{n}}\right\|=0$. Let $a \in A$ such that its stabilizer in $\Gamma_{0}$ is finite. The last part of the proof of (1) implies that $\lim _{n \rightarrow \infty} \sup _{\gamma \in X}\left\|\pi(\rho(\gamma)(a))\left(\xi_{k_{n}}\right)-\xi_{k_{n}}\right\|=0$. On the other hand, since the stabilizer of $a$ in $\Gamma_{0}$ is finite and $\pi$ is a $c_{0}$ representation, we get that

$$
\lim _{\gamma \rightarrow \infty}\left\langle\pi(\rho(\gamma)(a))\left(\xi_{n}\right), \xi_{n}\right\rangle=0 .
$$

Altogether, this gives a contradiction, as $X$ is infinite.

3.2 Remarks. (a) We note that the proof of part (1) in fact shows more: if $X \subset A$ is a set such that the inclusion $\left(X \subset A \rtimes_{\rho} \Gamma_{0}\right)$ has relative property $(\mathrm{T})$, then the inclusion $\left(X \subset A \rtimes_{\tilde{\rho}} \Gamma\right)$ has relative property (T). 
(b) Let us also remark that the proof of (2) can be adapted to show that if $A \rtimes_{\rho} \Gamma_{0}$ is not Haagerup, then $A \rtimes_{\tilde{\rho}} \Gamma$ is not Haagerup, provided that the stabilizer of some $a \in A$ in $\Gamma_{0}$ is finite. Indeed, in the notation from above, if $A \rtimes_{\rho} \Gamma_{0}$ is not Haagerup, then we can find an infinite set $X \subset A \rtimes_{\rho} \Gamma_{0}$ and a sequence $\left\{k_{n}\right\}_{n \geq 1}$ such that $\lim _{n \rightarrow \infty} \sup _{\gamma \in X}\left\|\sigma_{k_{n}}(\gamma)\left(\eta_{k_{n}}\right)-\eta_{k_{n}}\right\|=0$. Thus, $\lim _{n \rightarrow \infty} \sup _{\gamma \in X}\left\|\sigma_{k_{n}}\left(\gamma a \gamma^{-1}\right)\left(\eta_{k_{n}}\right)-\eta_{k_{n}}\right\|=0$. If the projection of $X$ onto $\Gamma_{0}$ is infinite, then $\left\{\gamma a \gamma^{-1} \mid \gamma \in X\right\}$ is an infinite subset of $A$ and a contradiction is reached as in the above proof. Otherwise, the set $Y$ of all $a \in A$ such that $(a, \gamma) \in X$, for some $\gamma \in \Gamma_{0}$, is infinite. Since the projection of $X$ onto $\Gamma_{0}$ is finite, it is clear that $\lim _{n \rightarrow \infty} \sup _{a \in Y}\left\|\sigma_{k_{n}}(a)\left(\eta_{k_{n}}\right)-\eta_{k_{n}}\right\|=0$. Again, we obtain a contradiction as in the end of the proof of part (1).

Recently, Y. de Cornulier, Y. Stalder and A. Valette have shown that if two countable groups $A$ and $\Gamma$ have Haagerup's property, then so does their wreath product $A<\Gamma=A^{\Gamma} \rtimes \Gamma$ ([SSV9]). More generally, they consider generalized wreath product groups $A \imath_{X} \Gamma=A^{X} \rtimes \Gamma$, where $X$ is a countable $\Gamma$-set and $\Gamma$ acts on $A^{X}=\bigoplus_{x \in X} A$ by shifting indices. In this context, they show that if $A$ and $\Gamma$ are Haagerup, then so are certain wreath products $A l_{X} \Gamma$ (e.g. when $X$ is a Haagerup quotient group of $\Gamma$ ) ([CSV09, Theorem 6.2]). Furthermore, it is conjectured in CSV09 that all such wreath products are Haagerup, regardless of the set $X$. As a consequence of Theorem 3.1, we disprove this conjecture by showing, for example, that if $X$ is an infinite quotient of $\Gamma$ with property $(\mathrm{T})$, then $A{ }_{{ }_{X}} \Gamma$ is not Haagerup.

3.3 Corollary. Let $A$ be a non-trivial countable group. Let $\Gamma$ be a countable group together with a quotient group $\Gamma_{0}$. Endow $\Gamma_{0}$ with the left multiplication action of $\Gamma$. Then the generalized wreath product $A{ }_{\Gamma_{0}} \Gamma$ is Haagerup if and only if $A, \Gamma$ and $\Gamma_{0}$ are Haagerup.

Proof. The if part is a particular case of CSV09, Theorem 6.2]. To prove the only if part, assume that $A 2_{\Gamma_{0}} \Gamma$ is Haagerup. Since both $A$ and $\Gamma$ are subgroups of $A\left\{_{\Gamma_{0}} \Gamma\right.$, they must be Haagerup. Let $a \in A \backslash\{e\}$ and let $A_{0}$ be the cyclic group generated by $a$. Since $A_{0} \imath_{\Gamma_{0}} \Gamma$ is Haagerup (being a subgroup of $A 2_{\Gamma_{0}} \Gamma$ ) and $A_{0}$ is abelian, Theorem 3.1 (2) implies that $\Gamma_{0}$ is Haagerup.

The above corollary gives new examples of countable groups which are not Haagerup and yet do not admit any infinite subgroups with relative property $(\mathrm{T})$. More precisely, in the above context, $G=A \tau_{\Gamma_{0}} \Gamma$ is such a group, whenever $A, \Gamma$ are Haagerup and $\Gamma_{0}$ is not. Indeed, by Corollary 3.3, $G$ is not Haagerup, while by [CSV09, Theorem 6.7] (or by [Io07, Theorem 3.6]), $G$ does not have relative property $(\mathrm{T})$ with respect to any infinite subgroup. Moreover, we can use results from Po06a], Po06b] and [Io07] to deduce that the group von Neumann algebra of $G$ has no rigid diffuse von Neumann subalgebra:

3.4 Corollary. Let $A$ be a non-trivial countable Haagerup group. Let $\Gamma$ be a countable Haagerup together with a quotient group $\Gamma_{0}$. Assume that $\Gamma_{0}$ is not Haagerup and endow it with the left multiplication action of $\Gamma$. Then the group von Neumann algebra $N=L\left(A \tau_{\Gamma_{0}} \Gamma\right)$ does not have Haagerup property and does not admit any diffuse von Neumann subalgebra $B$ such that the inclusion $(B \subset N)$ is rigid.

Proof. By Corollary 3.3, $A 2_{\Gamma_{0}} \Gamma$ does not have Haagerup's property, and therefore its group von Neumann algebra does not have it either (see e.g. Po06a]). Now, 
assume by contradiction that the inclusion $(B \subset N)$ is rigid, for some diffuse von Neumann subalgebra $B$ of $N$. Since $\Gamma$ has Haagerup's property, the proof of [P006a, Theorem 6.2] gives that a corner of $B$ embeds into $L\left(A^{\Gamma_{0}}\right)$, in the sense of [Po06b, Section 2].

To get a contradiction, we apply results from [Io07, leaving the details to the reader. Notice first that $N$ can be written as $\left(\bar{\bigotimes}_{\Gamma_{0}} L(A)\right) \rtimes \Gamma$, where $\Gamma$ acts on $\bar{\bigotimes}_{\Gamma_{0}} L(A)$ by Bernoulli shifts. Using this observation and the rigidity of the inclusion $(B \subset N)$, the proof of [Io07, Theorem 3.6] implies that a corner of $B$ can be embedded into $L\left(A^{F}\right)$, for some finite subset $F$ of $\Gamma_{0}$. Finally, the proof of Io07, Corollary 3.7] shows that since $L(A)$ has Haagerup's property, $B$ cannot be diffuse, a contradiction.

\section{ACKNOWLEDGMENTS}

The authors are grateful to Professors Sorin Popa and Yehuda Shalom for useful discussions and encouragement.

\section{REFERENCES}

[Bu91] M. Burger: Kazhdan constants for SL(3,Z), J. Reine Angew. Math. 413 (1991), 3667. MR.1089795 (92c:22013)

[Ch83] M. Choda: Group factors of the Haagerup type, Proc. Japan Acad. 59 (1983), 174177. MR718798 (85f:46117)

[CJ85] A. Connes, V.F.R. Jones: Property (T) for von Neumann algebras, Bull. London Math. Soc. 17 (1985), 57-62. MR766450(86a:46083)

[CCJJV01] P.A. Cherix, M. Cowling, P. Jolissaint, P. Julg, A. Valette: Groups with the Haagerup Property, Birkhäuser, Progress in Mathematics 197, 2001. MR:1852148(2002h:22007)

[Co06a] Y. de Cornulier: Kazhdan and Haagerup properties in algebraic groups over local fields, J. Lie Theory 16 (2006), 67-82. MR2196414(2006i:22018)

[Co06b] Y. de Cornulier: Relative Kazhdan property, Ann. Sci. Ecole Norm. Sup. 39 (2), (2006), 301-333. MR.2245534 (2007c:22008)

[CSV09] Y. de Cornulier, Y. Stalder, A. Valette: Proper actions of wreath products and generalizations, preprint arXiv:0905.3960.

[Fu09] A. Furman: A survey of Measured Group Theory, preprint arXiv:0901.0678.

[GHW05] E. Guenter, N. Higson, S. Weiberger: The Novikov conjecture for linear groups, Publ. Math. Inst. Hautes Études Sci. No. 101 (2005), 243-268. MR.2217050 (2007c:19007)

[Io07] A. Ioana: Rigidity results for wreath product II 1 factors, Journal of Functional Analysis 252 (2007), 763-791. MR.2360936 (2008j:46046)

[Io09] A. Ioana: Relative Property (T) for the Subequivalence Relations Induced by the Action of $S L_{2}(\mathbb{Z})$ on $\mathbb{T}^{2}$, Adv. Math. 224 (2010), no. 4, 1589-1617. MR2646305

[Ka67] D. Kazhdan: On the connection of the dual space of a group with the structure of its closed subgroups, Funct. Anal. and its Appl. 1 (1967), 63-65. MR0209390 (35:288)

[Ma82] G. Margulis: Finitely-additive invariant measures on Euclidian spaces, Ergodic Theory Dynam. Systems 2 (1982), 383-396. MR721730 (85g:28004)

[Ma91] G. Margulis: Discrete subgroups of semisimple Lie groups, Springer, 1991. MR 1090825 (92h:22021)

[MvN36] F.J. Murray, J. Von Neumann: On rings of operators, Ann. of Math. (2) 37 (1936), no. 1, 116-229. MR 1503275

[Pe09] J. Peterson: Examples of group actions which are virtually $W^{*}$-superrigid, preprint 2009.

[Po06a] S. Popa: On a class of type $I I_{1}$ factors with Betti numbers invariants, Ann. of Math. (2) 163 (2006), no. 3, 809-899. MR 2215135 (2006k:46097)

[Po06b] S. Popa: Strong rigidity of $I I_{1}$ factors arising from malleable actions of w-rigid groups I, Invent. Math. 165 (2006), 369-408. MR2231961 (2007f:46058) 
[Po07] S. Popa: Deformation and rigidity for group actions and von Neumann algebras, International Congress of Mathematicians. Vol. I, 445-477, Eur. Math. Soc., Zürich, 2007. MR 2334200 (2008k:46186)

[Wi08] D. Witte Morris: Introduction to Arithmetic Groups, lecture notes, available at http://people.uleth.ca/ dave.morris/.

Department of Mathematics, 1326 Stevenson Center, Vanderbilt University, Nashville, Tennessee 37240 - and - Institute of Mathematics of the Romanian Academy, Bucharest, Romania

E-mail address: ionut.chifan@vanderbilt.edu

Department of Mathematics, University of California, los Angeles, los Angeles, California 90095-155505 - And - Institute of Mathematics of the Romanian Academy, Bucharest, Romania

E-mail address: adiioana@math.ucla.edu 